Laboratories for Cell Biology, Rheumatology and Experimental Oncology,

Medical School, University of São Paulo,

Brazil

\title{
Differential Staining of Collagens Type I, II and III by Sirius Red and Polarization Microscopy
}

\author{
L. C. U. Junqueira, W. Cossermelli and R. Brentani
}

Received November, 8, 1977

\begin{abstract}
Summary. Organs of fish, amphibian, reptile, bird and mammals when stained by Sirius Red and studied with polarization microscopy present different colors in regions where collagens I, II and III have been described.

Collagen type I presented a yellow, orange or red color while collagen type III appeared green. Collagen type II, present in cartilage and chondrosarcoma showed a variable color according to the tissue and the species. Its color and morphology however always permitted its clear distinction from collagens type I and type III.
\end{abstract}

In 1964 Sweat, Puchtler and Rosenthal searching for a substituent of van Gieson's method that would stain collagen permanently, introduced Sirius Red in histological technique. Four years later Constantine and Mowry (1968) observed that this stain increased the birefrigency of collagen fibers when studid with polarization microscopy.

Recent biochemical studies show that at least four types of collagen can be found in the organs of vertebrates. Collagen type $\mathrm{I}$ is the main component in adult skin dermis, bone, tendon and dentin. Collagen II is the major or sole component in cartilage and chondrosarcoma. Type III is usually found associated with type $I$ in the aorta, uterus, leiomyoma and fetal dermis (for a review on the distribution of collagen types see Miller, 1976). Recently it was also observed in the peripheral nerve (Seyer, KAng and Whitaker, 1977). Collagen type IV is present in basement membranes.

We present here some results obtained using the Sirius Red stain followed by polarization microscopy to study the presence of collagen types I, II and III in different tissues and organs. As will be seen, our results strongly suggest that this method permits the distinction of these collagen types in vertebrate tissues.

\section{Materials and Methods}

The following species of vertebrates were used: Tilapia melanopleura, and Geophagus brasiliensis (fish), Bufo ictericus (amphibian), Xenodon merremii and Phylodrias schotii (reptiles), Gallus gallus (bird) and mouse, rat, guinea pig, rabbit and dog. At least three specimens of each species were studied. The experimental animals were anesthetised with barbiturate.

Human material was collected from autopsies of three young normal adult males who died in traffic accidents. Organs were fixed in Bouin's fluid at room temperature for $5-24$ hrs. Bone was decalcified in 10\% aqueous EDTA. After routine paraffin embedding $5 \mu \mathrm{m}$, sections were made and stained for $30 \mathrm{~min}$ in the Picrosirius 


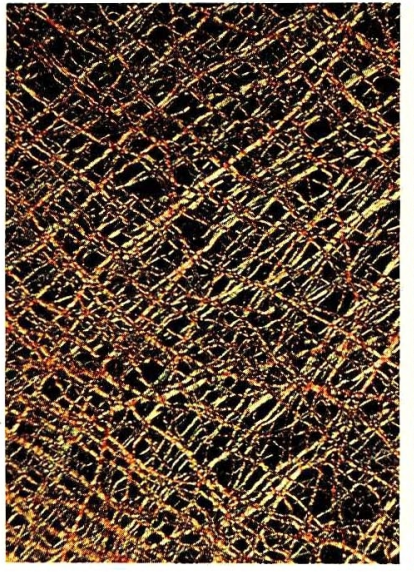

1

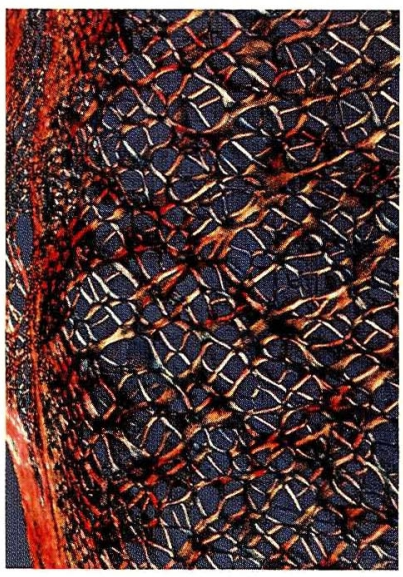

4

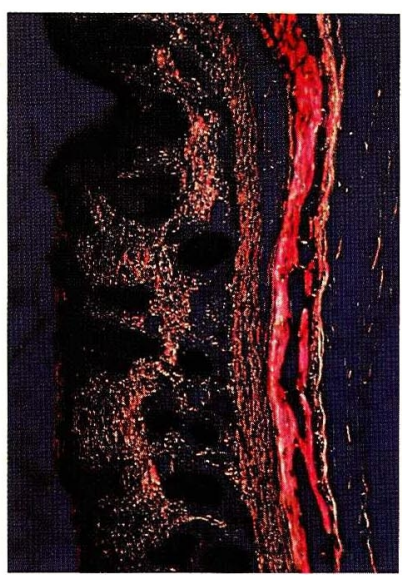

7

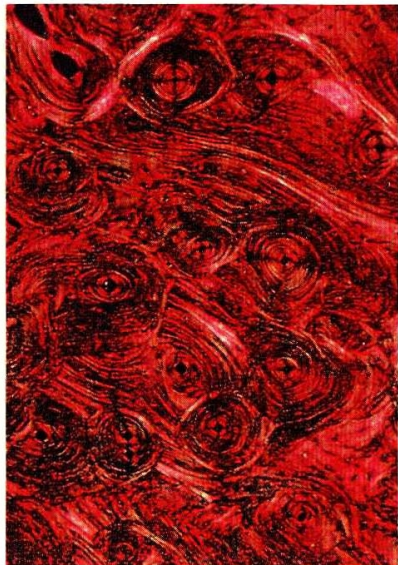

2

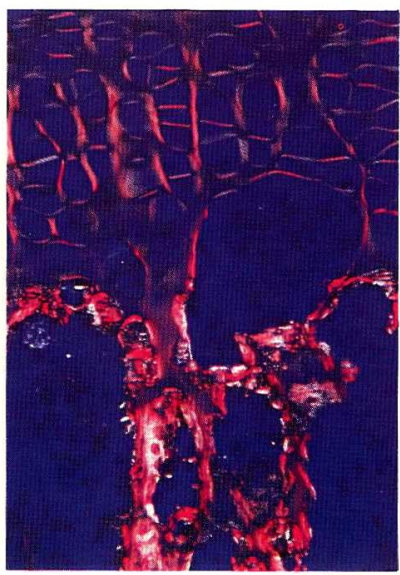

5

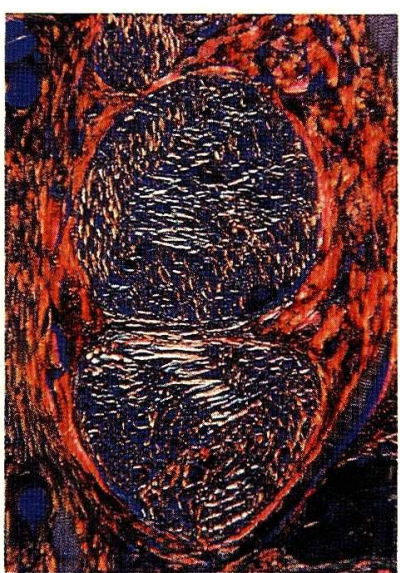

8

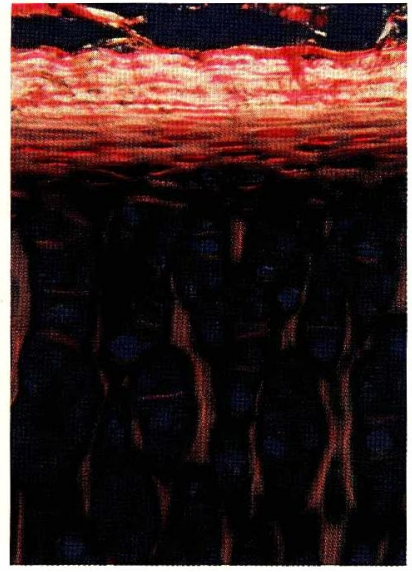

3

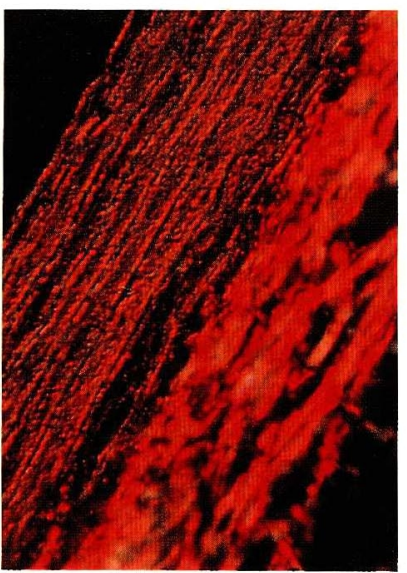

6

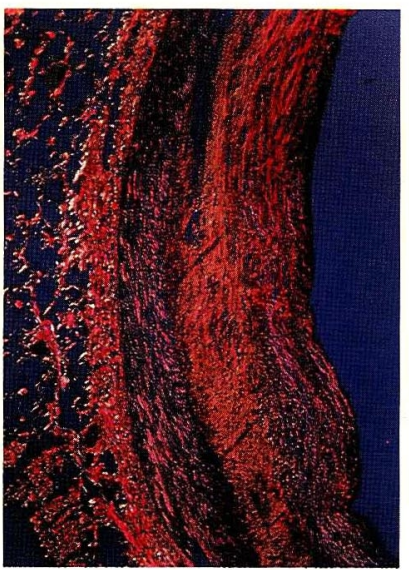

9

Fig. 1-9. Legends in opposite page. 
solution described by Sweat, Puchtler and Rosenthal (1964), i.e., 0.1\% Sirius Red F3BA (from Verona Dyestuffs: P. O. Box 385: Springfield Road: Union; New Jersey) in satured aqueous picric acid.

The sections were washed in running water, dehydrated in alcohol, cleared in xylene and mounted in synthetic resin. Observations were performed with a Nikon model LKE microscope using crossed polarizing filters. For the study of collagens type II and III it is essential to use a very intense light. This was obtained by overloading the voltage of the illumination lamp.

Photography was performed using a Pentax K2 camera and Kodak Ektachrome $\mathrm{X}$ or Kodacolor II films. As the colors obtained depended on the color filters used and lamp voltage, whenever possible, comparison was made between sections observed under the same conditions.

To compare the different colors observed with the Picrosirius stain plus polarization microscopy, $35 \mathrm{~mm}$ positive photomicrographs from Ektachrome $\mathrm{X}$ film, were taken under standardized conditions. From these photographs areas containing the image of different types of collagen were cut out, inserted in a cuvette and their absorption spectra were obtained using a model 25 Beckmann's registering spectrophotometer.

\section{Results}

Collagen type I. In all animals studied, the collagen from adult skin, bone, dentin, capsule and binding connective tissue from various organs and tendon presents a brilliant yellow, orange or red color when stained with Picrosirius and observed with crossed polaroid filters (Fig. 1, 2). All following descriptions will refer to observations realized under these conditions. Bone from all species and dermis of some species tended to present a darker hue more on the orange or red side. Tendons however tended toward a lighter color. Perichondrium and the connective capsule or envelope of various organs such as salivary glands, kidney, liver, testicles, striated

All figures from sections stained in Picrosirius and photographed with crossed polarizing filters.

Fig. 1. Rat mesentery. Total preparation. Bright yellow color in the collagen fibers. $\times 150$

Fig. 2. Bone of human rib. Red color in collagen type I. $\times 150$

Fig. 3. Rabbit tracheal cartilage. Observe the yellow bright color in the perichondrium (collagen type I) and as paler brownish color in the collagen from the cartilage (collagen type II). A transition zone is clearly visible between the perichondrium and the cartilage. $\times 300$

Fig. 4. Sternal cartilage of the frog Bufo ictericus. At the left the perichondrium with red fibers. The disposition of the cartilage collagen fibers is clearly visible in this type of preparation. $\times 120$

Fig. 5. Ossification in a rat femur epiphyseal plate. In the upper region the cartilage. Below red fibers of newly forming bone. $\times 130$

Fig. 6. Rat aorta. Observe the adventitial tunic (right) presenting red thick collagen fibers. In the left side the media and intima with thin green fibers. $\times 300$

Fig. 7. Section of newborn rat skin. To the right the hypodermis with red stained collagen fibers. In the left side just below the epidermis a region with thin and pale green fibers. $\times 50$

Fig. 8. Cross section of rat sciatic nerve. Observe thick yellow fibers in the perineurium. The endoneurium is formed by thin fibers with a greenish hue. $\times 120$

Fig. 9. Cross section of the coronary artery of a patient with atherosclerosis. Compare with Fig. 6. The intima and media present a large amount of thick yellow collagen fibers. $\times 120$ 
muscle and peripheral nerves presented a bright orange or red color in all species studied (Fig. 3, 8).

Collagen type II. The following observations refer mainly to tracheal cartilage, tibio-femoral articular cartilage, sternal cartilage and chondrosarcoma. In all species studied the collagen of the cartilage could be distinctly seen presenting a different color from the one seen in the perichondrium, bone or dermis. Frequently two distinct colors could be seen according to the collagen orientation. In one direction a blue color could be seen while in collagen disposed at $90^{\circ}$ from the blue, a light yellow color coud be seen. The exact colors varied considerably in their hues according to the cartilage and the species. However, in all cases, the color presented by the cartilage could be clearly distinguished from the color of collagen type I containing structures (Fig. 3, 4). In cartilages with a perichondrium (such as in the trachea and sternum) a clear transition zone could be observed between the cartilage and the perichondrium (Fig. 3). This zone might represent a region, where collagens type I and II coexist. The distinction between collagens I and II is also clear in endochondral ossification observed in the ephiphyseal plate cartilage of long bones of the species studied. In this situation the color of the collagen from the cartilages contrasts vigorously against the red or orange color of the newly formed bone trabeculae (Fig. 5).

In transplantable rat chondrosarcoma, a tumor known to contain collagen type II (SмIтн et al., 1975), results similar to those in cartilages were obtained: the capsule showed a bright yellow color and the cartilage, blue and pale yellow colors. The colors presented by collagen type II were much less intense than observed with collagen type I and were only clearly visible when very intense illumination was used. As exceptionally sharp images of collagen distribution could be obtained in the cartilages studied we feel that this method might be very useful to study the architecture of this tissue.

Collagen type III. This type of collagen was described in the aorta, uterus, leiomyoma fetal skin and peripheral nerves.

Contrary to collagens types I and II which are present as major components in different tissues, in all structures above mentioned, collagen type III is known to be mixed with an appreciable amount of collagens type $I$. It is therefore difficult to get direct data as to the color presented by collagen type III.

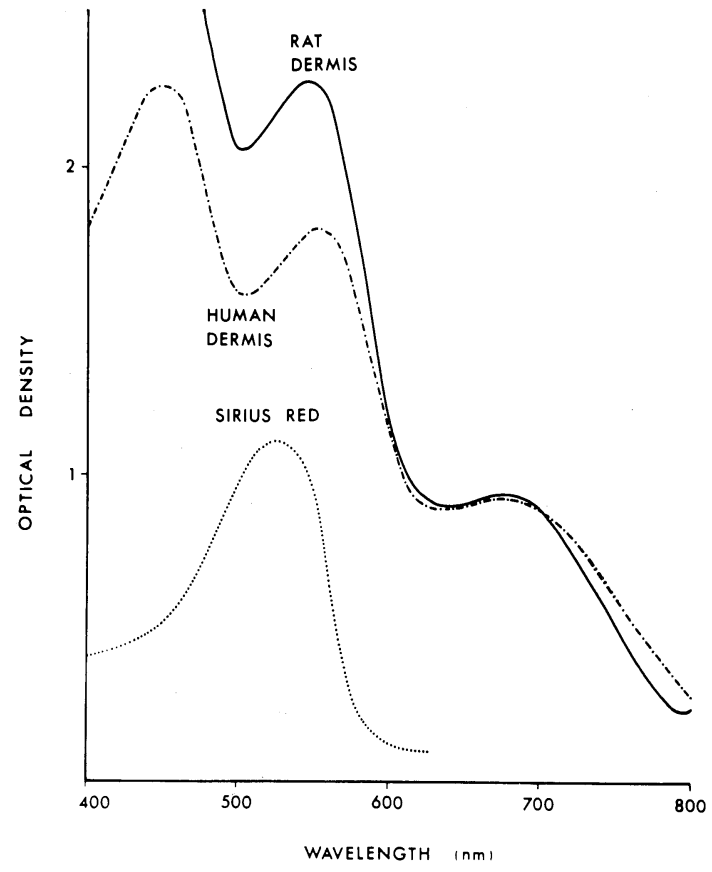

Fig. 10. Absorption spectra from color photomicrographs of human and rat dermis and from an aqueous solution of Sirius Red. 
The following observations however support the idea that collagen III presents a green color in tissue sections. Thus in all collagen type III containing organs from the various species studied, invariably regions containing thin green fibers could be observed beside regions with thick bright yellow fibers. This green color is much less intense than the yellow and is observable only when very intense illumination is used.

In the aorta, arteries and large arterioles of all species studied, two distinct layers could be distinguished. A peripheral region corresponding to the tunica adventitia of this blood vessel contained coarse bright yellow or red collagen fibers. The inner layer (corresponding to the tunica media plus tunica intima, presents thin, distinctly green fibers (Fig. 6).

In the uterus and leiomyoma distinct regions of thicker yellow fibers alternate with regions in which thin green fibers predominate. In leiomyomas these alternating regions are more accentuated and frequently form whorls.

In newborn rats the dermis showed two distinct layers. The layer just below the epidermis was formed by thin green fibers, while the deep layer presented thick yellow fibers (Fig. 7). In adult rats and all other mammals studied, only thick bright yellow or red fibers formed a thick layer in the dermis.

We suspected the presence of collagen III in nerves due to the observation of thin green fibers present mainly in the central region of these structures. However, the connective tissue of the epineurium always presents itself bright yellow or red (Fig. 8). These observations agree with the recent results from SAYER, KANG and WHITAKER (1977) showing with a biochemical method, the coexistence of collagens type I and III in human peripheral nerves.

All these observations that coincide with the data from the biochemical literature (see discussion) lead us to believe that the thick yellow fibers are collagen type I, while the green thin fibers represent collagen type III.

Thin green fibers were also observed in the smooth muscle layers of the digestive tract in all species studied. The histological aspect suggests that these smooth muscle layers must be a rich source of collagen III.

Recently, an increase in the content of collagen type I as compared to collagen type III has been described in the tunica intima of the aorta of humans with atherosclerosis (McCullagh and Balian,

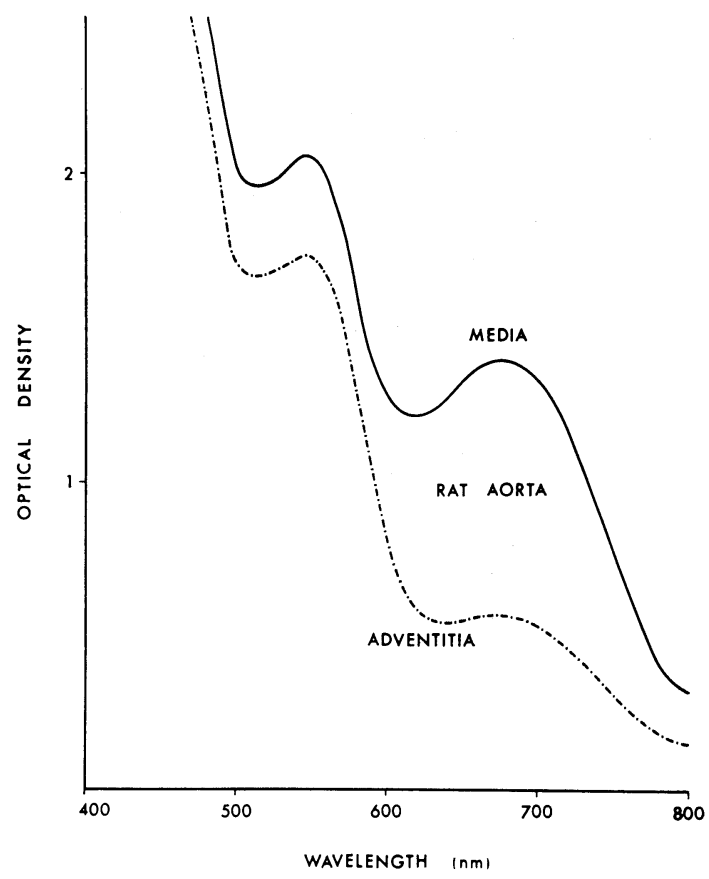

Fig. 11. Absorption spectra from the medial and adventitial tunics from a rat aorta. The collagen III containing region presents a peak at $700 \mathrm{~nm}$. 
1975). The study of material from eight autopsies of advanced cases of human atherosclerosis showed that the tunica media and tunica intima of the arteries and arterioles that normally present thin green fibers are invaded by thick bright yellow collagen fibers characteristic of collagen type I (Fig. 9). This will be the subject of a separate paper in preparation.

Spectrophotometric analysis of the color photomicrographs. The analysis of the absorption spectra of color photomicrographs of structures containing collagen type I such as adult rat and human dermis, aorta's adventitial tunic and perichondrium showed similar spectra as seen in Figures 10 and 11. Observe that the curves have a low peak around $700 \mathrm{~nm}$ and a high peak close to $550 \mathrm{~nm}$. This $550 \mathrm{~nm}$ peak is probably due to Sirius Red as pure solutions of this substance present a peak at approximately the same wavelength (Fig. 10).

The absorption spectrum from the tunic media of the aorta that presents a green color in the microscope (Fig. 6) showed beside the $550 \mathrm{~nm}$ peak a characteristic high absorption around $700 \mathrm{~nm}$.

This peak was also observed in the tunica media of the aorta of other animals and reflects its typical green color. Absorption spectra from cartilage micrographs gave us variable results confirming our visual observations.

\section{Discussion}

We feel that our results strongly suggest that the Picrosirius stain associated with polarization microscopy can distinguish between collagens types I, II and III.

The consistent bright yellow, orange or red colors observed in structures containing exclusively collagen type I such as bone, dermis, dentin and tendon speaks strongly for these colors being specific to this type of collagen.

The collagen of cartilage and chondrosarcoma, structures known to contain only collagen type II (SMiтh et al., 1975; MILLER, 1976) presented variable colors. However, these colors are always distinct from the ones observed in collagens type I and III.

The difference between collagens type I and II is particularly striking in zones where they are present side by side as in the superficial region of tracheal cartilage or in endochondral ossification.

Our observations comparing the color of the collagen of the perichondrium with the collagen of cartilage agree with the results of vON DER MARK and VON DER MARK (1977) who observed with the immunohistochemical method the presence of collagen type I in the perichondrium and collagen type II in the cartilage.

A precise characterization of collagen type III is however, more difficult because it is always associated with collagen type I. In all cases described in the biochemical literature, where collagen type III coexists with collagen type I such as in the aorta (Trelstad, 1974), fetal dermis (Epstein, 1974), leiomyoma (Chung and Miller, 1974) and peripheral nerves (SEYER, KANG and Whitaker, 1977), the presence of thick yellow or red fibers in certain regions was observed associated with portions of the tissue that presented predominantly thin green fibers. There is therefore, a coincidence between the presence of thin green fibers and collagen type III, in the organs mentioned above in all species studied. These green fibers, by the way, are not visible in tissues that have only collagen type I. 
Our results with the aorta are comparable to those obtained by GAy et al. (1975) who, using the immunohistochemical method, described collagen type $I$ in the tunica adventitia and collagen type III in the tunica media and tunica intima. We feel that the immunohistochemical method, being highly specific, lends strong support to the hypothesis that collagen type III presents a green color with the method used.

The extremely consistent and uniform results of our comparative studies in representative species of the main orders of vertebrates strongly suggests uniformity of collagen distribution and biology in these animals.

The spectral analysis results of the colors in the photomicrographs support our visual observations in the microscope in the sense that the regions containing thin green fibers presumed to be of collagen type III present a characteristic peak at $700 \mathrm{~nm}$. It is interesting to observe that all spectra studied always present the $550 \mathrm{~nm}$ peak characteristics of Sirius Red.

The mechanism and conditions of Picrosirius staining are being studied in this laboratory and will be the subject of a future publication.

We feel that the Picrosirius-polarization method should be further explored and has a potential usefulness in the study of the biology and pathology of connective tissue.

\section{References}

Constantine, V. S. and R. W. Mowry.: Selective staining of human dermal collagen II. The use of Picrosirius Red F3BA with polarization microscopy. J. invest. Dermatol. 50: 414-419 (1968).

Chung, F. and E. J. Miller.: Collagen polymorphism: Characterization of molecules with the chain composition $\left[\alpha_{1}(\mathrm{III})\right]_{3}$ in human tissues. Science 183: 1200-1201 (1974).

Epstein, E. H. Jr.: $\quad\left[\alpha_{1}(\mathrm{III})\right]_{3}$ human skin collagen. Release by pepsin digestion and preponderance in fetal life. J. biol. Chem. 249: 3225-3232 (1974).

Gay, S., L. Balleisen, K. Remberger, P. P. Fietzek, B. C. Adelman and K. Kühn : Immunohistochemical evidence for the presence of collagen type III in human arterial walls, arterial thrombi and in leukocytes incubated with collagen in vitro. Klin. Wochenschr. 53: 899-902 (1975).

Mark, K. von der and $\mathbf{H}$. von der Mark. : Immunological and biochemical studies of collagen type transition during in vitro chondrogenesis of chick limb mesodermal cells. J. Cell Biol. 73: 736-747 (1977).

McCullagh, K. A. and G. Balian. : Collagen characterization and cell transformation in human atherosclerosis. Nature (Lond.) 258: 73-75 (1975).

Miller, E. J. : Biochemical characteristics and biological significance of the genetically distinct collagens. Molec. cell. Biochem. 13: 165-192 (1976).

Seyer, J. M., A. H. Kang and J. N. Whitaker : The caracterization of Type I and Type III of collagens from human peripheral nerve. Biochim. biophys. Acta 492: 415-425 (1977).

Smith, B. D., G. R. Martin, E. J. Miller, A. Dorfman and R. S. Swarm : Nature of collagen synthetized by a transplanted chondrosarcoma. Arch. Biochem. Biophys. 166: 181-186 (1975).

Sweat, F., H. Puchtler and S. I. Rosenthal : Sirius Red F3BA as a stain for connective tissue. Arch. Pathol. 78: 69-72 (1964). 
Trelstad, R. L.: Human aorta collagens: evidence for three distinct species. Biochem. biophys. Res. Commun. 57: 717-725 (1974).

Prof. L. C. U. Junqueira

Faculdade de Medicina USP

Caixa Postal 2921

0.1000 São Paulo, S. P.

Brasil 